\title{
BiP Inducer X: An ER Stress Inhibitor for Enhancing Recombinant Antibody Production in $\mathrm{CHO}$ Cell Culture
}

\author{
Kwang Ha, Tae; Hansen, Anders H; Kildegaard, Helene Faustrup; Min Lee, Gyun
}

\section{Published in:}

Biotechnology Journal

Link to article, DOI:

10.1002/biot.201900130

Publication date:

2019

Document Version

Peer reviewed version

Link back to DTU Orbit

Citation (APA):

Kwang Ha, T., Hansen, A. H., Kildegaard, H. F., \& Min Lee, G. (2019). BiP Inducer X: An ER Stress Inhibitor for Enhancing Recombinant Antibody Production in CHO Cell Culture. Biotechnology Journal, [e1900130]. https://doi.org/10.1002/biot.201900130

\section{General rights}

Copyright and moral rights for the publications made accessible in the public portal are retained by the authors and/or other copyright owners and it is a condition of accessing publications that users recognise and abide by the legal requirements associated with these rights.

- Users may download and print one copy of any publication from the public portal for the purpose of private study or research.

- You may not further distribute the material or use it for any profit-making activity or commercial gain

- You may freely distribute the URL identifying the publication in the public portal 
Gyun Min Lee ORCID iD: 0000-0002-9876-7378

Bip inducer X: an ER stress inhibitor for enhancing recombinant antibody production in $\mathrm{CHO}$ cell culture

Tae Kwang $\mathrm{Ha}^{1}$, Anders Holmgaard Hansen ${ }^{1}$, Helene Faustrup Kildegaard ${ }^{1}$, Gyun Min Lee ${ }^{1,2}$

${ }^{1}$ The Novo Nordisk Foundation Center for Biosustainability, Technical University of Denmark, Kgs. Lyngby, 2800, Denmark

${ }^{2}$ Department of Biological Sciences, KAIST, Daejeon, 34141, Republic of Korea

Correspondence: Prof. Gyun Min Lee, Department of Biological Sciences, KAIST, Daejeon, 34141, Republic of Korea

Email: gyunminlee@kaist.ac.kr

\section{Additional correspondence:}

Dr. Helene Faustrup Kildegaard, The Novo Nordisk Foundation Center for Biosustainability, Technical University of Denmark, Kgs. Lyngby, 2800, Denmark

Email: hef@biosustain.dtu.dk

Keywords: $\mathrm{BiP}$ inducer $\mathrm{X}, \mathrm{CHO}$ cells, ER stress inhibitor, galactosylation, monoclonal antibody

This article has been accepted for publication and undergone full peer review but has not been through the copyediting, typesetting, pagination and proofreading process, which may lead to differences between this version and the Version of Record. Please cite this article as doi: 10.1002/biot.201900130. 
Abbreviations: ATF6, activating transcription factor 6; B4galt, $\beta-1,4-$ galactosyltransferase; BiP, binding immunoglobulin protein; BIX, BiP inducer $\mathrm{X}$; CHO, Chinese hamster ovary; CHOP, C/EBP homologous protein; DMSO, dimethyl sulfoxide; eIF2, eukaryotic initiation factor 2; ER, endoplasmic reticulum; Gla, galactosidase $\alpha$; Glb1, galactosidase $\beta 1$; GRP94, heat shock protein 90 beta family member 1; GS, glutamine synthetase; IVCC, time integral of viable cell concentration; mAb, monoclonal antibody; MMC, maximum mAb concentration; MSX, methionine sulfoximine; MVCC, maximum viable cell concentration; NaBu, sodium butyrate; $\boldsymbol{q}_{\mathbf{m A b}}$, specific mAb productivity; TUDCA, tauroursodeoxycholic acid; $\boldsymbol{\mu}$, specific growth rate; UPR, unfolded protein response; Xbp1, X-box binding protein

\begin{abstract}
Prolonged endoplasmic reticulum (ER) stress reduces protein synthesis and induces apoptosis in mammalian cells. When dimethyl sulfoxide (DMSO), a specific monoclonal antibody productivity $\left(q_{\mathrm{mAb}}\right)$-enhancing reagent, was added to recombinant Chinese hamster ovary (rCHO) cell cultures (GSR cell line), it induced ER stress and apoptosis in a dose-dependent manner. To determine an effective ER stress inhibitor, three ER stress inhibitors (BiP inducer X (BIX), tauroursodeoxycholic acid, and carbazole) were examined, and BIX showed the best production performance. Co-addition of BIX (50 $\mu \mathrm{M})$ with DMSO extended the culture longevity and enhanced $q_{\mathrm{mAb}}$. As a result, the maximum mAb concentration was significantly increased with improved galactosylation. Co-addition of BIX significantly increased the expression level of $\mathrm{BiP}$ followed by increased expression of chaperones (calnexin and GRP94) and galactosyltransferase. Furthermore, the expression levels of CHOP, a well-known ER stress marker, and cleaved caspase-3 were significantly reduced, suggesting that BIX addition reduced ER stress-induced cell death by relieving ER stress. The beneficial effect of BIX on mAb production was also demonstrated with another $q_{\mathrm{mAb}}$-enhancing reagent (sodium butyrate) and a different rCHO cell line
\end{abstract}


(CS13-1.00). Taken together, BIX is an effective ER stress inhibitor that can be used to increase mAb production in rCHO cells.

\section{Graphical Abstract}

Endoplasmic reticulum (ER) stress is induced in CHO cell culture, especially with the addition of productivity-enhancing chemicals. BIX is an effective ER stress inhibitor for use in recombinant $\mathrm{CHO}$ cell cultures for improved mAb production.

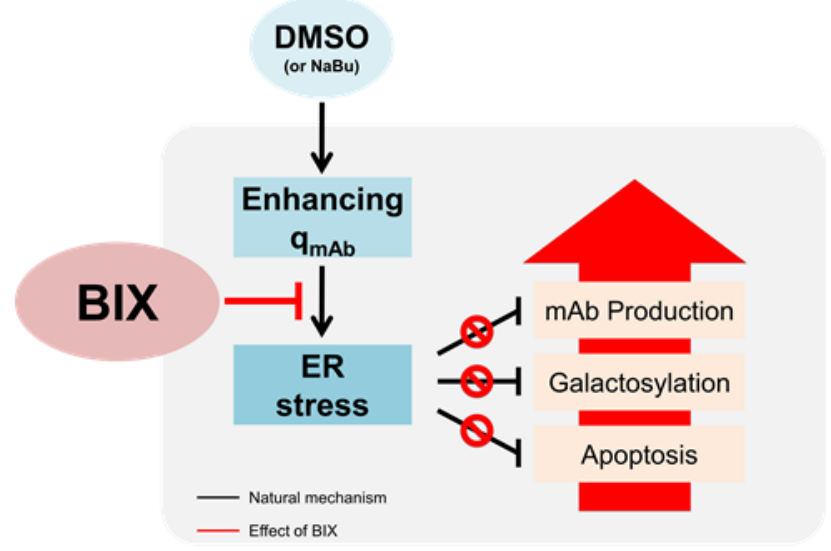

\section{Introduction}

Chinese hamster ovary (CHO) cells are commonly used for industrial production of monoclonal antibodies (mAbs). Over the last two decades, increased demand for mAbs has led to the need to increase the expression level of high quality mAbs in recombinant $\mathrm{CHO}$ (rCHO) cells.

There have been several reports on the post-translational bottlenecks in the biosynthetic pathway of therapeutic proteins in $\mathrm{rCHO}$ cells $[1,2]$, which suggest that the expression level of mAbs can be increased by improving the secretory pathway machinery in rCHO cells. Endoplasmic reticulum (ER), a central organelle of the 
secretory pathways, plays a role in regulating protein translocation, protein synthesis, and early post-translational modifications, including folding and glycosylation $[3,4]$. In mammalian cells, highly increased expression of target proteins activates ER stress [5,6]. Chemical additives such as dimethyl sulfoxide (DMSO) and sodium butyrate $(\mathrm{NaBu})$, which have been used for enhancing mAb production in $\mathrm{rCHO}$ cell cultures [7-10], may also activate ER stress. DMSO has been observed to induce ER stress in mouse embryonic cells [11]. When cells are exposed to ER stress, an unfolded protein response (UPR) is activated to restore stress or to adapt to stress conditions in order to maintain homeostasis and cellular function $[12,13]$. However, prolonged ER stress reduces protein synthesis to minimize ER load and ultimately induces mitochondrial dysfuntion/depolarization leading to apoptotic cell death [4,13].

To cope with ER stress and cell death induced by ER stress, genetic manipulationsrelated to proteins that affect ER capacity and function, such as spliced X-box binding protein (Xbp1s), signaling receptor proteins, and molecular chaperones, have been attempted in $\mathrm{CHO}$ cells, resulting in enhanced production of therapeutic proteins including mAbs [14-19]. Alternatively, chemical ER stress inhibitors may also be used in rCHO cell cultures. A chemical approach for reducing ER stress is easy to implement in industrial processes due to its simplicity. ER stress inhibitors such as BiP inducer X (BIX), tauroursodeoxycholic acid (TUDCA), and carbazole have been used in various cell lines including neuroblastoma, hepatoblastoma, and pheochromocytoma lines [20-23]. These ER stress inhibitors protected cells from ER stress and improved cell viability through regulation of ER stress-related genes and proteins. However, despite the potential of such ER stress inhibitors, they have never been used as a means of enhancing the expression level of mAbs in rCHO cell cultures.

This article is protected by copyright. All rights reserved. 
In this study, to find an effective ER stress inhibitor for rCHO cell cultures, three different ER stress inhibitors, including BIX, were evaluated as chemical supplements for rCHO cells producing mAb. Of these, BIX showed the best effect on culture performance, and was added to rCHO cell cultures under ER stress induced by DMSO addition. The ER stress level and UPR levels were measured to understand the effect of BIX on mAb production and quality. Finally, BIX was evaluated as a chemical additive in fed-batch cultures under ER stress induced by DMSO addition.

\section{Experimental Section}

\subsection{Cell Line and Cell Maintenance}

The rCHO cell lines producing Rituximab (GSR) and a chimeric antibody (CS131.00) were used in this study. The GSR cell line was established through transfection of light and heavy chain vector encoding glutamine synthetase (GS) into GS knockout CHO-K1 cells as described previously [24]. Cells were selected at $25 \mu \mathrm{M}$ methionine sulfoximine (MSX, Sigma-Aldrich, St. Louis, MO). The CS13-1.00 cell line was established through transfection of light and heavy chain vectors encoding dihydrofolate reductase into CHO DG44 cells as described previously [25]. Cells were selected at $1 \mu \mathrm{M}$ methotrexate (MTX, Sigma-Aldrich). Both cell lines were adapted to grow in a serum-free suspension culture. Cells were maintained in $125 \mathrm{~mL}$ Erlenmeyer flasks (Corning, Corning, NY) with $30 \mathrm{~mL}$ of PowerCHO2CD (Lonza, Basel, Switzerland) supplemented with GSEM (Sigma-Aldrich) and $25 \mu \mathrm{M}$ MSX for the GSR cell line or with $1 \mu \mathrm{M}$ MTX for the CS13-1.00 cell line in a humidified shaking incubator at $120 \mathrm{rpm}, 37^{\circ} \mathrm{C}$, and $5 \% \mathrm{CO}_{2}$.

This article is protected by copyright. All rights reserved. 


\subsection{ER Stress Inhibitor Screening}

GSR cells were inoculated at $3 \times 10^{5}$ cells $/ \mathrm{mL}$ into 6 -well plates with $3 \mathrm{~mL}$ of culture medium and the plates were incubated in a humidified shaking incubator at $120 \mathrm{rpm}$, $37^{\circ} \mathrm{C}$, and $5 \% \mathrm{CO}_{2}$. BIX, TUDCA, and carbazole were dissolved in distilled water at a concentration of 10,50 , and $5 \mathrm{mM}$, respectively. After 3 days of cultivation, each ER stress inhibitor was individually added to the cultures at various concentrations. As controls, cell cultures without any supplements were performed. All chemicals were purchased from Sigma-Aldrich, unless otherwise noted. Samples were harvested every two days to measure the viable cell concentration, viability, and mAb concentration. Culture supernatants were aliquoted and stored at $-70^{\circ} \mathrm{C}$ for further analysis.

\subsection{Shake Flask Culture}

Cells were inoculated at $3 \times 10^{5}$ cells/mL into $125 \mathrm{~mL}$ Erlenmeyer flasks with $30 \mathrm{~mL}$ of culture medium and incubated in a humidified shaking incubator at $120 \mathrm{rpm}, 37^{\circ} \mathrm{C}$, and $5 \% \mathrm{CO}_{2}$. After 3 days of cultivation, $50 \mu \mathrm{M}$ BIX and/or $1 \% \mathrm{v} / \mathrm{v}$ DMSO was added to the cultures of GSR cells or CS13-1.00 cells. As controls, cell cultures without any supplements were also performed. Samples were collected daily for measuring the viable cell concentration, viability, and mAb concentration. Culture supernatants were aliquoted and stored at $-70^{\circ} \mathrm{C}$ for further analysis.

\subsection{Bioreactor Culture}

Cells were inoculated at $2.5 \times 10^{5}$ cells/mL into a bioreactor (Eppendorf DASGIP, Jülich, Germany) with a $270 \mathrm{~mL}$ working volume in the absence or presence of 50 $\mu \mathrm{M}$ BIX and/or $1 \% \mathrm{v} / \mathrm{v}$ DMSO on day 3. Cultures were maintained at $37^{\circ} \mathrm{C}$, $\mathrm{pH} 7.15$, 
$50 \%$ air saturation, and an agitation speed of $200 \mathrm{rpm}$. For fed-batch cultures, CHO CD EfficientFeed ${ }^{\mathrm{TM}} \mathrm{B}$ (Invitrogen, Carlsbad, CA) was added to the cultures daily from day 4 to 8 at a $5 \%$ v/v ratio. Glucose was also added to the cultures daily to adjust the concentration to $24 \mathrm{mM}$. Samples were taken daily to determine the viable cell concentration and for further analyses.

\subsection{Viable Cell Concentration and mAb Concentration}

Viable cell concentration was measured using a NucleoCounter NC-200 cell counter (ChemoMetec, Allerod, Denmark). The mAb concentration was estimated using an Octet RED96 (Pall, Menlo Park, CA) [26]. Specific mAb productivity ( $\left.q_{\mathrm{mAb}}\right)$ was evaluated from a plot of the mAb concentration against the time integral values of the viable cell concentration [27].

\subsection{Western Blot Analysis}

Western blot analysis was performed as described previously [26]. The antibodies used for western blot analysis were anti-GRP78/BiP, CHOP, cleaved caspase-3, ATF6, XBP1s, eIF2 $\alpha$, p-eIF2 $\alpha$, GRP94, and calnexin. Anti-vinculin was used as a loading control. All antibodies were purchased from Cell Signaling (Cell Signaling Technology, Beverly, MA).

\subsection{Quantitative Real Time Polymerase Chain Reaction (qRT-PCR)}

The qRT-PCR was run on an Mx3005P (Agilent Technologies, Santa Clara, CA) using Brilliant III Ultra-Fast SYBR1 Green master mix (Agilent Technologies) as described previously [26]. The target genes used for qRT-PCR were categorized into

(1) ER stress-related genes: GRP78/BiP and CHOP and (2) galactosylation-related 
genes: B4galt1, B4galt2, B4galt3, Gla, and Glb1. GAPDH was used as an internal control.

\subsection{Purification and Glycan Analysis of mAb}

Cell culture supernatants were harvested from the flask and bioreactor cultures. Methods for purification and glycan analysis of mAbs were described previously [28]. Briefly, mAbs contained in the supernatants were purified by protein A affinity chromatography (recombinant protein A agarose, Pierce, Rockford, IL), according to the manufacturer's instructions. N-glycans of mAbs were labeled with GlykoPrep Rapid N-Glycan kit (ProZyme, Hayward, CA), according to the manufacturer's instructions. N-glycan analysis was performed using a Thermo Ultimate 3000 HPLC system coupled with Thermo Velos Pro Iontrap MS (Thermo Fisher Scientific, Waltham, MA).

\subsection{Statistical Analysis}

Reported values are expressed as mean \pm standard deviation, unless otherwise noted. The data were analyzed using a two tailed Student's $t$-test. The differences between means were considered significant at $P<0.05$.

\section{Results}

\subsection{BIX Was the Best ER Stress Inhibitor for Improved mAb Production}

To find an effective ER stress inhibitor for rCHO cell cultures, three ER stress inhibitors at various concentrations (10 and $50 \mu \mathrm{M}$ BIX, 50 and $200 \mu \mathrm{M}$ TUDCA, and 1 and $5 \mu \mathrm{M}$ carbazole) were examined. The rCHO cell lines producing Rituximab (GSR) cells were cultivated in six-well plates and each ER stress inhibitor was 
individually added to the cultures on day 3. Cells were also cultivated without any supplement as controls. The concentration of each ER stress inhibitor used in this study was determined according to the literature reports [20-23]. Experiments were performed three times.

Figure 1 shows the profiles of cell growth, viability, and mAb concentration during the cultures. Compared to the control cultures, all ER stress inhibitors suppressed cell growth, but to a different extent (Fig. 1A). However, cells in cultures with BIX addition were viable for a longer period (Fig. 1B). Despite suppressed cell growth, addition of $50 \mu \mathrm{M}$ BIX significantly increased the mAb production (Fig. 1C). The maximum mAb concentration (MMC) in the cultures with addition of $50 \mu \mathrm{M}$ BIX was $256.6 \pm 14.4 \mu \mathrm{g} / \mathrm{mL}$, which was approximately 1.6 times higher than that in the control cultures $(P<0.05)$. The time integral of viable cell concentration (IVCC) of the culture with addition of $50 \mu \mathrm{M}$ BIX was similar to that of control cultures due to the extended culture period. Thus, the highest MMC in cultures with $50 \mu \mathrm{M}$ BIX suggests that specific mAb productivity $\left(q_{\mathrm{mAb}}\right)$ with BIX was significantly higher than that in the control cultures. The $q_{\mathrm{mAb}}$ with $50 \mu \mathrm{M}$ BIX, which was calculated based on the data collected during the exponential growth phase, was $18.7 \pm 1.7 \mathrm{pg} / \mathrm{cell} / \mathrm{day}$, which was approximately 1.8 times higher than that in the control cultures. BIX was thus chosen as an ER stress inhibitor for improved mAb production.

\subsection{BIX Improved mAb Production in rCHO Cell Cultures Under DMSO- Induced ER Stress}

As observed in mouse embryonic cells, DMSO, which is known to increase the $q_{\mathrm{mAb}}$ of rCHO cells, may induce ER stress. To confirm this hypothesis, exponentially growing GSR cells in shake flasks were subjected to DMSO treatment at various 
concentrations $(0.5,1$, and $3 \% \mathrm{v} / \mathrm{v})$. DMSO inhibited cell growth but increased $q_{\mathrm{mAb}}$ in a dose dependent manner (Supplementary Fig. S1A-C). Although the highest $q_{\mathrm{mAb}}$ was obtained at 3\% DMSO, the highest MMC $(644.8 \pm 53.2 \mu \mathrm{g} / \mathrm{mL})$ was obtained at 1\% DMSO. The detrimental effect of 3\% DMSO on cell growth outweighed its beneficial effect on mAb production. DMSO induced ER stress in rCHO cells. ER stress and apoptotic cell death were significantly increased with increasing DMSO concentrations (Supplementary Fig. S1D).

To investigate whether BIX further improves mAb production under ER stressinduced culture conditions, GSR cells were cultivated in shake flasks with co-addition of $1 \%$ DMSO and $50 \mu \mathrm{M}$ BIX on day 3. As controls, cells were cultivated without any supplements. For comparison, cells were also cultivated with the addition of BIX or DMSO alone on day 3. Cultures were performed three times.

Figure 2 shows the profiles of cell growth, viability, and mAb concentration during the cultures. As observed in 6-well plate cultures, addition of BIX only slightly suppressed cell growth and prolonged culture duration (Fig. 2A and 2B). The maximum viable cell concentration (MVCC) and specific growth rate ( $\mu$ ) in the control cultures $\left(4.75 \pm 0.13 \times 10^{6}\right.$ cells $/ \mathrm{mL}$ and $0.52 \pm 0.04$ day $\left.^{-1}\right)$ decreased to 4.37 $\pm 0.24 \times 10^{6}$ cells $/ \mathrm{mL}$ and $0.50 \pm 0.03$ day $^{-1}$ in the culture with addition of BIX only, respectively. However, the IVCC of cultures with the addition of BIX only was higher than that in control cultures due to the extended culture period. In contrast, addition of DMSO only rapidly decreased cell viability in the decline phase of growth (Fig. 2A and 2B). However, when BIX was added to the cultures with DMSO, the negative effect of DMSO on cell viability was alleviated and the culture longevity was extended by 3 days.

This article is protected by copyright. All rights reserved. 
Regardless of the single or combined addition of BIX and DMSO, $q_{\mathrm{mAb}}$ was significantly increased. Thus, despite depressed cell growth, the MMC in the cultures with the addition of DMSO only was $525.3 \pm 96.2 \mu \mathrm{g} / \mathrm{mL}$, which is approximately 4.6 times higher than that in the control cultures. The highest $q_{\mathrm{mAb}}$ was obtained in cultures with the co-addition of BIX and DMSO, which was 4.3 times higher than that in the control cultures. As a result, due to both enhanced $q_{\mathrm{mAb}}$ and extended culture longevity, the MMC in cultures with co-addition of BIX and DMSO was $1096.3 \pm$ $77.3 \mu \mathrm{g} / \mathrm{mL}$, which is 10.0 times higher than that in the control cultures (Fig. 2C). The $\mu$, MVCC, $q_{\mathrm{mAb}}$, MMC, and IVCC in the cultures shown in Fig. 2 are summarized in Table 1.

\subsection{BIX Reduced DMSO-Induced ER Stress, UPR, and Apoptosis}

DMSO significantly increased the $q_{\mathrm{mAb}}$, but also induced severe ER stress and apoptotic cell death (supplementary Fig. S1). BIX is known to directly reduce ER stress by inducing $\mathrm{BiP}$ expression, which is one of the main factors for the prosurvival pathway under ER stress [24]. To understand the positive effect of BIX on DMSO-induced ER stress, cells were sampled in the cultures shown in Fig. 2 and the levels of mRNA and proteins related to ER stress and apoptosis were measured by qRT-PCR and western blot, respectively.

Figure $3 \mathrm{~A}$ and $3 \mathrm{~B}$ show the mRNA expression levels of two major pathway genes for ER stress, BiP as a pro-survival signal and CHOP as a pro-apoptotic cell death signal. GAPDH was used as a control gene. In control cultures, the expression levels of both $\mathrm{BiP}$ and $\mathrm{CHOP}$ increased during the cultures, suggesting that ER stress accumulated during the cell cultures (Fig. 3A and 3B). Addition of DMSO alone significantly increased the expression level of $\mathrm{BiP}$ and $\mathrm{CHOP}$ during the cultures. Co- 
addition of BIX and DMSO further increased the expression level of BiP (Fig. 3A). However, the expression level of CHOP was significantly decreased, demonstrating the potential of BIX as an ER stress inhibitor (Fig. 3B).

Figure 3C shows the western blots of Bip, CHOP, and cleaved-caspase-3. Changes in the protein expression levels of Bip and CHOP during the cultures were consistent with those in the mRNA expression levels. When BIX and DMSO were co-added to the cultures, the protein expression level of CHOP, unlike Bip, was significantly decreased. Likewise, co-addition of BIX and DMSO significantly decreased the protein expression level of cleaved-caspase-3 that is a well-known marker for apoptosis [12.13]. Thus, inhibition of ER stress by BIX reduced apoptotic cell death.

To determine the effect of BIX on the UPR and chaperone activation under DMSOinduced ER stress, the expression level of proteins involved in these pathways was measured by western blotting. Figure 4 show the western blots of proteins related to the UPR and chaperones. Vinculin was used as a loading control. In the control cultures, the expression levels of UPR-related proteins (ATF6, XBP1s, p-eIF2 $\alpha$, and eIF2 $\alpha$ ) and chaperones (Calnexin and GRP94) were increased during the cultures, as observed with those of BiP and CHOP shown in Fig. 3B. Addition of DMSO only significantly increased the expression level of these proteins. When both DMSO and BIX were added to the cultures, the expression levels of these proteins were increased further. Particularly, the expression level of ATF6 on day 8 was 3.7 and 1.4 times higher than that in the control cultures and the cultures with addition of DMSO only, respectively. Likewise, the expression level of GRP94 on day 8 was 3.9 and 1.7 times higher than that in the control cultures and the cultures with addition of DMSO only, respectively. Thus, co-addition of DMSO and BIX activated the UPR pathway 
followed by an increase in chaperone expression, which may be responsible for enhanced $q_{\mathrm{mAb}}$ without severe cell death.

\subsection{Co-Addition of BIX and DMSO Improved Galacotsylation of mAb}

The highest MMC was obtained in cultures with the co-addition of BIX and DMSO (Fig. 2C). To determine the effect of BIX and DMSO on galactosylation of mAbs, culture supernatants were harvested on day 6 and 9 and mAbs were purified for glycan analyses. Figure 5A shows the profiles of the galactosylated glycan proportion of mAbs. In control cultures, the G0 form increased from $57.9 \pm 5.1 \%$ on day 6 to $61.0 \pm 1.4 \%$ on day 9 with a concomitant decrease in G1 and G2 forms, but it was not statistically significant. In cultures with the addition of BIX and DMSO, the G0 form was much smaller than that in the control cultures. In addition, the G0, G1, and G2 forms did not change significantly from day 6 to day 9 in the cultures with co-addition of BIX and DMSO. The G1 and G2 forms on day 9 were $46.6 \pm 0.8$ and $11.0 \pm 1.3$, respectively, which were significantly higher than those in the control cultures $(P<$ 0.01 and $P<0.05)$.

To understand the beneficial effect of BIX and DMSO on galactosylation, the mRNA expression levels of galactosylation-related genes (galactosyltransferases: B4galt1, 2, and 3, and galactosidases: Gla and Glb1) were measured by qRT-PCR. Figure 5B shows the mRNA expression profiles of these genes during the cultures. Overall, the mRNA expression levels of galactosyltransferases in cultures with BIX and DMSO addition were higher than those in the control cultures. On day 4, the mRNA expression levels of B4galt1 and 3, which are known to play a dominant role in galactosylation in $\mathrm{CHO}$ cells, were significantly higher compared to those in the control cultures $(P<0.05)$. In contrast, the mRNA expression levels of galactosidases 
in the culture with co-addition of BIX and DMSO were not significantly higher than those in the control cultures $(P>0.05)$. Thus, the increased expression level of galactosyltransferase genes by the addition of BIX and DMSO contributed in part to the improved galactosylation of mAbs.

\subsection{Co-Addition of BIX and DMSO Improved mAb Production in Fed-Batch}

\section{Cultures}

To further determine the potential of BIX and DMSO for improving mAb production, GSR cells were cultivated in a fed batch mode in a bioreactor. BIX (50 $\mu \mathrm{M})$ and DMSO (1\%) were co-added to the cultures on day 3, followed by daily feeding of nutrient cocktails from day 5 to 10 . As controls, cells were also cultivated without BIX and DMSO. Cultures were performed two times.

Figure 6 shows the culture profiles of cell growth, viability, mAb concentration, and galactosylation of mAb during the fed-batch cultures. Culture profiles were plotted daily before feeding the nutrient cocktails, to avoid obtaining excessively complex profiles. As observed in the shake flask cultures (Fig. 2), co-addition of BIX and DMSO inhibited cell growth and extended the culture duration (Fig. 6A and 6B). Due to enhanced $q_{\mathrm{mAb}}$, the MMC in fed-batch cultures with BIX and DMSO (1109.8 \pm $156.9 \mu \mathrm{g} / \mathrm{mL}$ ) was 4.9 times higher than that in the control fed-batch cultures (Fig. 6C). Furthermore, the G1 and G2 forms of mAb in the fed-batch cultures with BIX and DMSO were significantly higher than that in the control fed-batch cultures $(P<$ 0.05) (Fig. 6D), demonstrating the potential of BIX and DMSO for improving mAb production in fed-batch cultures. The $\mu$, MVCC, $q_{\mathrm{mAb}}$, and MMC in the cultures shown in Fig. 6 are summarized in Table 2.

This article is protected by copyright. All rights reserved. 


\subsection{Co-Addition of BIX and DMSO Improved mAb Production in CS13-1.00 Cell}

\section{Cultures}

To generalize the beneficial effect of BIX and DMSO on mAb production, the same set of cell cultures in shake flasks were performed with CS13-1.00 cells. As observed in the cultures of GSR cells (Fig. 2), co-addition of BIX and DMSO inhibited cell growth and extended the culture longevity (Fig. 7A and 7B). Due to enhanced $q_{\mathrm{mAb}}$ and extended culture longevity, the MMC in cultures with co-addition of BIX and DMSO was approximately 2.3 and 1.5 times higher than that in the control cultures and the cultures with addition of DMSO alone, respectively (Fig. 7C). Furthermore, the G1 and G2 form of mAb in cultures with BIX and DMSO was significantly higher than that in the control cultures on day $9(P<0.05)$ (Fig. 7D). Thus, the addition of BIX and DMSO improved mAb production in rCHO cell cultures.

\section{Discussion}

When protein folding capacity is overwhelmed by stress inducers, mis/un-folded proteins are accumulated in the ER and induce ER stress [30,31]. Accumulated ER stress then triggers the UPR pathways to restore ER homeostasis. The UPR, an adaptive mechanism against ER stress, is regulated by three different sensor proteins: ATF6, IRE1 $\alpha$, and PERK. To maintain the function of cells, the three sensors play an important role in increasing the protein folding capacity by upregulation of chaperones, degradation of un/mis-folded proteins, and inhibition of translation $[12,13]$. However, if these adaptive responses are not sufficient to restore protein folding homeostasis, UPR signaling promotes apoptotic cell death mechanisms [4].

Since the chemical approach to reducing ER stress in rCHO cell cultures by medium supplementation is simple and easy to implement in industrial processes, we 
examined the effects of three different ER stress inhibitors (BIX, TUDCA, and carbazole) with rCHO cells producing mAbs. Among the three, only BIX showed a beneficial effect on cell viability and mAb production. BIX was screened to have the highest activity for BiP expression among approximately 10,000 compounds [20]. BIX is known to significantly reduce ER stress and apoptosis through increased BiP mRNA expression in various cell lines including neuroblastoma and retinal cells, and in animal models such as gerbils and mice [20,32,33].

$\mathrm{BiP}$, a molecular chaperone located in the ER lumen, binds to the three UPR sensor proteins and inactivates signal transduction under normal conditions. Under ER stress conditions, $\mathrm{BiP}$ is released from these complexes and redirects to the accumulated proteins to help protein folding and activate UPR signaling [29]. In addition, BiP itself interacts with the IgG assembly [34]. Previously, it was reported that $\mathrm{BiP}$ overexpression in $\mathrm{CHO}$ cells enhanced cell viability under serum deprivation and oxidative stress and increased antibody yields with reduced ER stress [14]. Cotransient transfection with $\mathrm{BiP}$ and the difficult to express antibody genes in $\mathrm{CHO}$ cells also increased the $q_{\mathrm{mAb}}$ [35]. In this study, BIX addition to rCHO cell cultures also significantly increased BiP expression and reduced apoptotic cell death, and thereby improved cell viability and MMC (Fig. 1 and 2).

Chemical additives such as DMSO and $\mathrm{NaBu}$ have been used for increasing recombinant protein production in $\mathrm{rCHO}$ cell cultures [7-10]. These chemical additives increased $q_{\mathrm{mAb}}$ but inhibited cell growth and induced apoptotic cell death [36,37]. In this study, DMSO addition was found to induce ER stress and apoptotic cell death in a dose-dependent manner (Supplementary Fig. 1). Like DMSO, NaBu addition also induced ER stress and apoptotic cell death [38]. Therefore, the beneficial 
effect of these chemical additives on $q_{\mathrm{mAb}}$ is compromised by their cytotoxic effect on cell growth.

Given that ER stress inhibitors like BIX can reduce ER stress and apoptosis induced by addition of $q_{\mathrm{mAb}}$-enhancing chemicals, combined addition of BIX and DMSO in rCHO cell cultures was hypothesized to further increase mAb production. As expected, co-addition of BIX and DMSO significantly increased the expression level of $\mathrm{BiP}$, while decreasing the expression level of CHOP (Fig. 3). CHOP, which inhibits the expression of anti-apoptotic genes, acts as a pro-apoptotic cell death signal induced by ER stress in neuronal cells, brain cells, and cancer cells like leukemia cells [39,40]. As a result, co-addition of BIX and DMSO significantly increased the MMC by reducing ER stress and apoptosis. Approximately 4.9 fold increase in MMC was achieved by the co-addition of BIX and DMSO in fed-batch cultures, which are widely used for large-scale commercial production of mAbs (Fig. 6). The synergistic effect of BIX and DMSO on mAb production was confirmed by performing the same set of experiments with another mAb producing $\mathrm{CHO}$ cell line (CS13-1.00). As observed in GSR cells, the co-addition of BIX and DMSO significantly improved mAb production by CS13-1.00 cells (Fig. 7). However, the extent of enhancement in mAb production was different probably because ER stress levels and stress responses differ between cell lines.

The beneficial effect of BIX on mAb production was further confirmed by performing the same set of experiments with $\mathrm{NaBu}$ (Supplementary Fig. 2). Addition of $1.5 \mathrm{mM} \mathrm{NaBu}$ only in GSR cell cultures inhibited cell growth and decreased cell viability in the decline phase of growth. Despite reduced cell growth, $\mathrm{NaBu}$ addition increased the MMC because of significantly enhanced $q_{\mathrm{mAb}}$. When BIX was added to 
the cultures with $\mathrm{NaBu}$, the negative effect of $\mathrm{NaBu}$ was relieved and culture longevity was extended by one day. As a result, the MMC in cultures with both BIX and $\mathrm{NaBu}$ was approximately 1.8 and 3.4-times higher than that in the cultures with only $\mathrm{NaBu}$ and control cultures, respectively. Thus, BIX can reduce ER stress and apoptosis induction by the addition of $q_{\mathrm{mAb}}$-enhancing chemicals, thus further enhancing mAb production.

In order to be used in rCHO cell cultures for mAb production, chemical additives should increase mAb production without negative impacts on mAb quality, for example on mAb glycosylation. However, some chemical additives such as $\mathrm{NaBu}$ have been reported to decrease galactosylation of mAbs in rCHO cell cultures [41]. It is well known that terminal galactosylation capping modulates complement dependent cytotoxicity (CDC) by affecting the binding of mAb to C1q in the complement system [42,43]. Recently, enhanced galactosylation of mAb has also been found to improve antibody-dependent cellular cytotoxicity (ADCC) [44]. However, co-addition of BIX and DMSO did not decrease the galactosylation of mAb in rCHO cell cultures but rather increased it (Fig. 5A). Furthermore, co-addition of BIX and DMSO also improved galactosylation of mAb in fed-batch culture where approximately 4.9-fold increase in MMC was achieved (Fig. 6). Since folding and quality control of secreted glycoproteins are regulated by UPR pathways in the ER, UPR activation, particularly Xbp1s, may enhance N-glycosylation of ER client proteins [45-47]. Xbp1s have been reported to modulate $\mathrm{N}$-glycan maturation pathways and to improve the synthesis of hybrid and complex N-glycans [48]. In this study, co-addition of BIX and DMSO significantly increased the expression level of Xbp1s, which may contribute in part to increase mAb galactosylation (Fig. 4). In addition, the increased expression level of 
galactosyltransferase genes by the addition of BIX and DMSO may also contribute in part to improved galactosylation of mAb.

In conclusion, ER stress was induced in $\mathrm{rCHO}$ cell culture, especially with the addition of $q_{\mathrm{mAb}}-$ enhancing chemicals such as DMSO and $\mathrm{NaBu}$, which negatively affected cell growth and mAb production in rCHO cell cultures. Among the three ER stress inhibitors tested in this study, BIX showed the best mAb production performance in rCHO cell cultures. Co-addition of BIX and DMSO significantly enhanced mAb production while increasing the galactosylated form of the mAb. Thus, BIX is an effective ER stress inhibitor for use in rCHO cell cultures for improved mAb production.

\section{Acknowledgement}

This research was supported by Danish Council for Independent Research Technology and Production Sciences (FTP), The Novo Nordisk Foundation (NNF10CC1016517), and the Ministry of Science, ICT and Future Planning for Basic Core Technology Development Program for the Oceans and the Polar Regions of the NRF (NRF-2016M1A5A1901813). The authors thank Helle Munck Petersen and Stefan Kol for technical assistance with the protein purification; Marianne Decker and Kristian Lund Jensen for technical assistance with the bioreactor experiments.

\section{Conflict of interest}

T. K. H. is listed as an inventor on a patent application based on this work. The remaining authors declare no financial or commercial conflict of interest.

This article is protected by copyright. All rights reserved. 


\section{References}

[1] S. Fischer, R. Handrick, K. Otte, The art of CHO cell engineering: A comprehensive retrospect and future perspectives. Biotechnol. Adv. 2015, 33, 18781896.

[2] D. Reinhart, W. Sommeregger, M. Debreczeny, E. Gludovacz, R. Kunert, In search of expression bottlenecks in recombinant CHO cell lines - A case study. Appl. Microbiol. Biotechnol. 2014, 98, 5959-5965.

[3] A. Ruggiano, O. Foresti, P. Carvalho, Quality control: ER-associated degradation: protein quality control and beyond. J. Cell Biol. 2014, 204, 869-879.

[4] R. Sano, J. C. Reed, ER stress-induced cell death mechanisms. Biochim. Biophys. Acta 2013, 1833, 3460-3470.

[5] Z. Du, D. Treiber, R. E. McCoy, A. K. Miller, M. Han, F. He, S. Domnitz, C. Heath, P. Reddy, Non-invasive UPR monitoring system and its applications in CHO production cultures. Biotechnol. Bioeng. 2013, 110, 2184-2194.

[6] K. Prashad, S. Mehra, Dynamics of unfolded protein response in recombinant CHO cells. Cytotechnology 2015, 67, 237-254.

[7] M. Fiore, R. Zanier, F. Degrassi, Reversible G(1) arrest by dimethyl sulfoxide as a new method to synchronize Chinese hamster cells. Mutagenesis 2002, 17, 419-424.

[8] L. Hunt, P. Batard, M. Jordan, F. M. Wurm, Fluorescent proteins in animal cells for process development: Optimization of sodium butyrate treatment as an example. Biotechnol. Bioeng. 2002, 77, 528-537.

[9] C. H. Liu, I. M. Chu, S. M. Hwang, Enhanced expression of various exogenous genes in recombinant Chinese hamster ovary cells in presence of dimethyl sulfoxide. Biotechnol. Lett. 2001, 23, 1641-1645.

[10] J. Rodriguez, M. Spearman, N. Huzel, M. Butler, Enhanced production of monomeric interferon-beta by $\mathrm{CHO}$ cells through the control of culture conditions. Biotechnol. Prog. 2005, 21, 22-30. 
[11] M. H. Kang, J. Das, S. Gurunathan, H. W. Park, H. Song, C. Park, J. H. Kim, The cytotoxic effects of dimethyl sulfoxide in mouse preimplantation embryos: a mechanistic study. Theranostics 2017, 7, 4735-4752.

[12] D. T. Rutkowski, R. J. Kaufman, A trip to the ER: coping with stress. Trends Cell Biol. 2004, 14, 20-28.

[13] M. Schröder, R. J. Kaufman, The mammalian unfolded protein response. Annu. Rev. Biochem. 2005, 74, 739-789.

[14] N. Borth, D. Mattanovich, R. Kunert, H. Katinger, Effect of increased expression of protein disulfide isomerase and heavy chain binding protein on antibody secretion in a recombinant CHO cell line. Biotechnol. Prog. 2005, 21, 106-111.

[15] K. Cain, S. Peters, H. Hailu, B. Sweeney, P. Stephens, J. Heads, K. Sarkar, A. Ventom, C. Page, A. Dickson, A CHO cell line engineered to express XBP1 and ERO1-L $\alpha$ has increased levels of transient protein expression. Biotechnol. Prog. 2013, 29, 697-706.

[16] J. Y. Chung, S. W. Lim, Y. J. Hong, S. O. Hwang, G. M. Lee, Effect of doxycycline-regulated calnexin and calreticulin expression on specific thrombopoietin productivity of recombinant Chinese hamster ovary cells. Biotechnol. Bioeng. 2004, 85, 539-546.

[17] S. O. Hwang, J. Y. Chung, G. M. Lee, Effect of doxycycline-regulated ERp57 expression on specific thrombopoietin productivity of recombinant $\mathrm{CHO}$ cells. Biotechnol. Prog. 2003, 19, 179-184.

[18] S. C. Ku, D. T. Ng, M. G. Yap, S. H. Chao, Effects of overexpression of X-box binding protein 1 on recombinant protein production in Chinese hamster ovary and NS0 myeloma cells. Biotechnol. Bioeng. 2008, 99, 155-164.

[19] H. Yoshida, S. Nadanaka, R. Sato, K. Mori, XBP1 is critical to protect cells from endoplasmic reticulum stress: evidence from Site-2 protease-deficient Chinese hamster ovary cells. Cell Struct. Funct. 2006, 31, 117-125.

This article is protected by copyright. All rights reserved. 
[20] T. Kudo, S. Kanemoto, H. Hara, N. Morimoto, T. Morihara, R. Kimura, T. Tabira, K. Imaizumi, M. Takeda, A molecular chaperone inducer protects neurons from ER stress. Cell Death Differ. 2008, 15, 364-375.

[21] Y. Y. Lee, S. H. Hong, Y. J. Lee, S. S. Chung, H. S. Jung, S. G. Park, K. S. Park, Tauroursodeoxycholate (TUDCA), chemical chaperone, enhances function of islets by reducing ER stress. Biochem. Biophys. Res. Commun. 2010, 397, 735-739.

[22] H. Miura, K. Takano, Y. Kitao, S. Hibino, T. Choshi, R. Murakami, H. Suzuki, M. Yamada, S. Ogawa, O. Hori, A carbazole derivative protects cells against endoplasmic reticulum (ER) stress and glutathione depletion. J. Pharmacol. Sci. 2008, 108, 164-171.

[23] M. G. Neuman, R. G. Cameron, N. H. Shear, S. Bellentani, C. Tiribelli, Effect of tauroursodeoxycholic and ursodeoxycholic acid on ethanol-induced cell injuries in the human Hep G2 cell line. Gastroenterology 1995, 109, 555-563.

[24] J. H. Park, S. M. Noh, J. R. Woo, J. W. Kim, G. M. Lee, Valeric acid induces cell cycle arrest at G1 phase in CHO cell cultures and improves recombinant antibody productivity. Biotechnol. J. 2016, 11, 487-496.

[25] S. J. Kim, N. S. Kim, C. J. Ryu, H. J. Hong, G. M. Lee, Characterization of chimeric antibody producing $\mathrm{CHO}$ cells in the course of dihydrofolate reductasemediated gene amplification and their stability in the absence of selective pressure. Biotechnol. Bioeng. 1998, 58, 73-84.

[26] T. B. Kallehauge, S. Li, L. E. Pedersen, T. K. Ha, D. Ley, M. R. Andersen, H. F. Kildegaard, G. M. Lee, N. E. Lewis, Ribosome profiling-guided depletion of an mRNA increases cell growth rate and protein secretion. Sci. Rep. 2017, 7, 40388.

[27] J. M. Renard, R. Spagnoli, C. Mazier, M. F. Salles, E. Mandine, Evidence that monoclonal antibody production kinetics is related to the integral of the viable cells curve in batch systems. Biotechnol. Lett. 1988, 10, 91-96.

[28] L. M. Grav, J. S. Lee, S. Gerling, T. B. Kallehauge, A. H. Hansen, S. Kol, G. M. Lee, L. E. Pedersen, H. F. Kildegaard, One-step generation of triple knockout CHO 
cell lines using CRISPR/Cas9 and fluorescent enrichment. Biotechnol. J. 2015, 10, 1446-1456.

[29] C. Hetz, S. Saxena, ER stress and the unfolded protein response in neurodegeneration. Nat. Rev. Neurol. 2017, 13, 477-491.

[30] A. H. Schönthal, Pharmacological targeting of endoplasmic reticulum stress signaling in cancer. Biochem. Pharmacol. 2013, 85, 653-666.

[31] H. Urra, E. Dufey, F. Lisbona, D. Rojas-Rivera, C. Hetz, When ER stress reaches a dead end. Biochim. Biophys. Acta 2013, 1833, 3507-3517.

[32] Y. Inokuchi, Y. Nakajima, M. Shimazawa, T. Kurita, M. Kubo, A. Saito, H. Sajiki, T. Kudo, M. Aihara, K. Imaizumi, M. Araie, H. Hara, Effect of an inducer of $\mathrm{BiP}$, a molecular chaperone, on endoplasmic reticulum (ER) stress-induced retinal cell death. Invest. Ophthalmol. Vis. Sci. 2009, 50, 334-344.

[33] Y. Oida, H. Izuta, A. Oyagi, M. Shimazawa, T. Kudo, K. Imaizumi, H. Hara, Induction of BiP, an ER-resident protein, prevents the neuronal death induced by transient forebrain ischemia in gerbil. Brain Res. 2008, 1208, 217-224.

[34] M. J. Feige, L. M. Hendershot, J. Buchner, How antibodies fold. Trends Biochem. Sci. 2010, 35, 189-198.

[35] L. P. Pybus, G. Dean, N. R. West, A. Smith, O. Daramola, R. Field, S. J. Wilkinson, D. C. James, Model-directed engineering of "difficult-to-express" monoclonal antibody production by Chinese hamster ovary cells. Biotechnol. Bioeng. 2014, 111, 372-385.

[36] T. K. Ha, A. H. Hansen, S. Kol, H. F. Kildegaard, G. M. Lee, Baicalein Reduces Oxidative Stress in CHO Cell Cultures and Improves Recombinant Antibody Productivity. Biotechnol. J. 2018, 13, e1700425.

[37] J. S. Lee, G. M. Lee, Effect of sodium butyrate on autophagy and apoptosis in Chinese hamster ovary cells. Biotechnol. Prog. 2012, 28, 349-357.

[38] J. Zhang, M. Yi, L. Zha, S. Chen, Z. Li, C. Li, M. Gong, H. Deng, X. Chu, J. Chen, Z. Zhang, L. Mao, S. Sun, Sodium Butyrate Induces Endoplasmic Reticulum 
Stress and Autophagy in Colorectal Cells: Implications for Apoptosis. PLoS One 2016, 11, e0147218.

[39] Y. Li, Y. Guo, J. Tang, J. Jiang, Z. Chen, New insights into the roles of CHOPinduced apoptosis in ER stress. Acta Biochim. Biophys. Sin. 2014, 46, 629-640.

[40] S. Oyadomari, M. Mori, Roles of CHOP/GADD153 in endoplasmic reticulum stress. Cell Death Differ. 2004, 11, 381-389.

[41] J. K. Hong, S. M. Lee, K. Y. Kim, G. M. Lee, Effect of sodium butyrate on the assembly, charge variants, and galactosylation of antibody produced in recombinant Chinese hamster ovary cells. Appl. Microbiol. Biotechnol. 2014, 98, 5417-5425.

[42] J. Hodoniczky, Y. Z. Zheng, D. C. James, Control of recombinant monoclonal antibody effector functions by Fc N-glycan remodeling in vitro. Biotechnol. Prog. 2005, 21, 1644-1652.

[43] T. S. Raju, Terminal sugars of Fc glycans influence antibody effector functions of IgGs. Curr. Opin. Immunol. 2008, 20, 471-478.

[44] M. Thomann, K. Reckermann, D. Reusch, J. Prasser, M. L. Tejada, Fcgalactosylation modulates antibody-dependent cellular cytotoxicity of therapeutic antibodies. Mol. Immunol. 2016, 73, 69-75.

[45] C. Hammond, I. Braakman, A. Helenius, Role of N-linked oligosaccharide recognition, glucose trimming, and calnexin in glycoprotein folding and quality control. Proc. Natl. Acad. Sci. 1994, 91, 913-917.

[46] E. D. Spear, D. T. Ng, Single, context-specific glycans can target misfolded glycoproteins for ER-associated degradation. J. Cell Biol. 2005, 169, 73-82.

[47] G. Thibault, N. Ismail, D. T. Ng, The unfolded protein response supports cellular robustness as a broad-spectrum compensatory pathway. Proc. Natl. Acad. Sci. 2011, 108, 20597-20602.

[48] M. B. Dewal, A. S. DiChiara, A. Antonopoulos, R. J. Taylor, C. J. Harmon, S. M. Haslam, A. Dell, M. D. Shoulders, XBP1s Links the Unfolded Protein Response 
to the Molecular Architecture of Mature N-Glycans. Chem. Biol. 2015, 22, 13011312.

\section{Legend of Tables}

Table 1 . The $\mu$, MVCC, $q_{\mathrm{mAb}}, \mathrm{MMC}$, and IVCC during shake flask cultures with the addition of BIX and/or DMSO. No supplements (C), $50 \mu \mathrm{M}$ BIX (T1), 1\% v/v DMSO (T2), and BIX and DMSO (T3). Values are means \pm standard deviations of three independent experiments.

Table 2. The $\mu$, MVCC, $q_{\mathrm{mAb}}, \mathrm{MMC}$, and IVCC during fed-batch cultures in a bioreactor with the addition of BIX and DMSO. No supplements (C), and BIX and DMSO (T). Values are means \pm standard deviations of duplicate experiments.

\section{Figures}

Figure 1. Profiles of (A) cell growth, (B) viability, and (C) mAb concentration in GSR cells during six-well plate cultures with various ER stress inhibitors. No inhibitor (black square), $10 \mu \mathrm{M}$ BIX (white circle), $50 \mu \mathrm{M}$ BIX (gray circle), $50 \mu \mathrm{M}$ TUDCA (white up-triangle), $200 \mu \mathrm{M}$ TUDCA (gray up-triangle), $1 \mu \mathrm{M}$ carbazole (white down-triangle), and $5 \mu \mathrm{M}$ carbazole (gray down-triangle). Error bars represent the standard deviations calculated from three independent experiments.

A

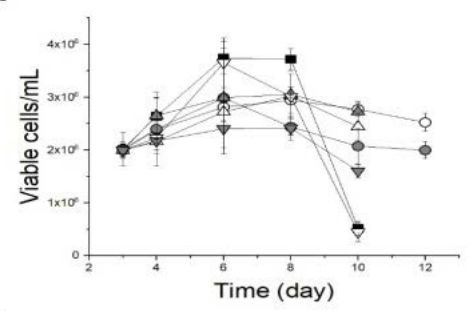

B

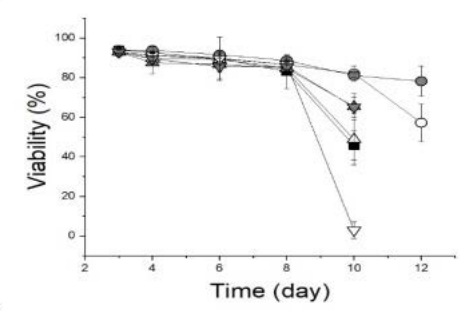

C

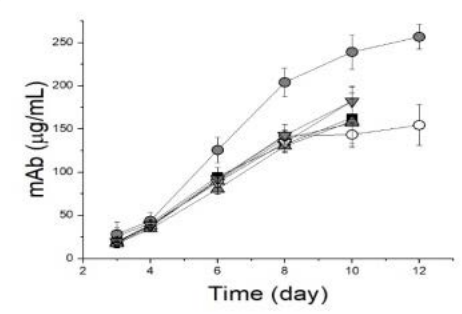

This article is protected by copyright. All rights reserved. 
Figure 2. Profiles of (A) cell growth, (B) viability, and (C) mAb concentration in GSR cells during shake flask cultures with the addition of BIX and/or DMSO. No chemical reagent (black square), $50 \mu \mathrm{M}$ BIX (black circle), 1 \% v/v DMSO (black uptriangle), and $50 \mu \mathrm{M}$ BIX and 1\% v/v DMSO (black down-triangle). Error bars represent the standard deviations calculated from three independent experiments.

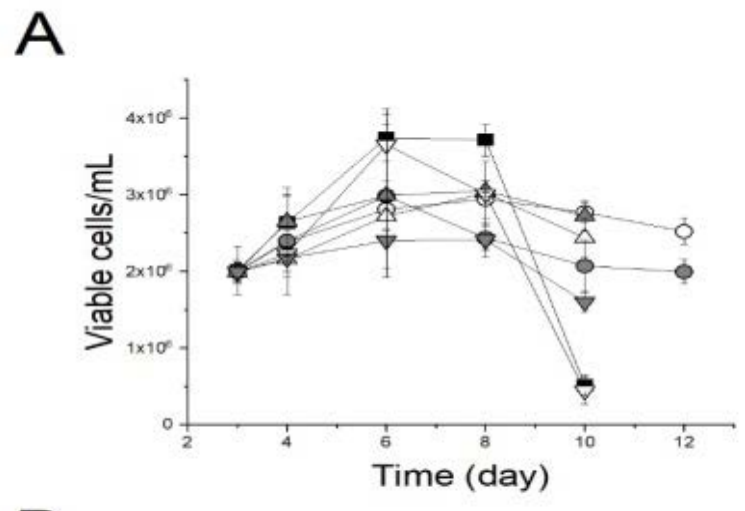

B

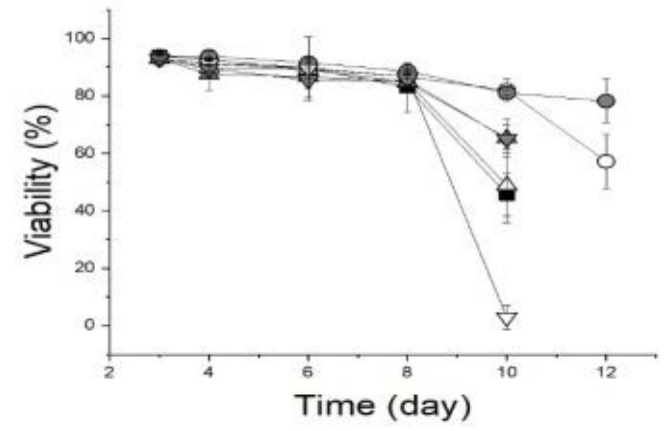

C

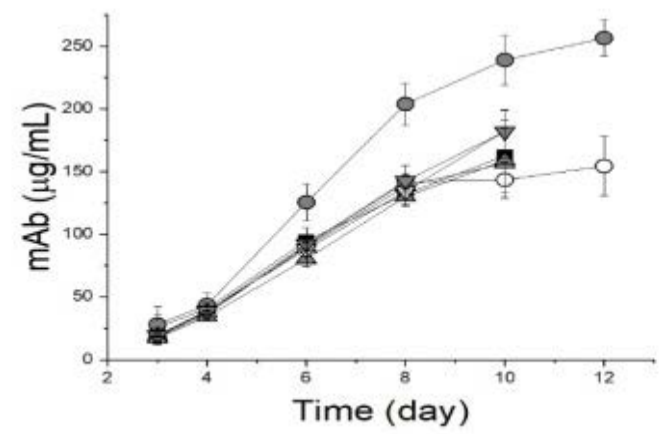

This article is protected by copyright. All rights reserved. 
Figure 3. (A) qRT-PCR of BiP and (B) CHOP during shake flask cultures. Cells were sampled on day 4, 6, and 8 of the cultures shown in Figure 2. Values were normalized to the control on day 4. No chemical reagent (white), BIX only (light gray), DMSO only (gray), and BIX and DMSO (black). Error bars represent the standard deviations calculated from three independent experiments. ${ }^{*} P<0.05$, ${ }^{* *} P<0.01$. (C) Western blots of BiP, CHOP, and cleaved caspase-3 during shake flask cultures. Cells were sampled on day 4, 6, and 8 of the cultures shown in Figure 2. Vinculin was used as a loading control. No chemical reagent (C), BIX only (T1), DMSO only (T2), and BIX and DMSO (T3).

A

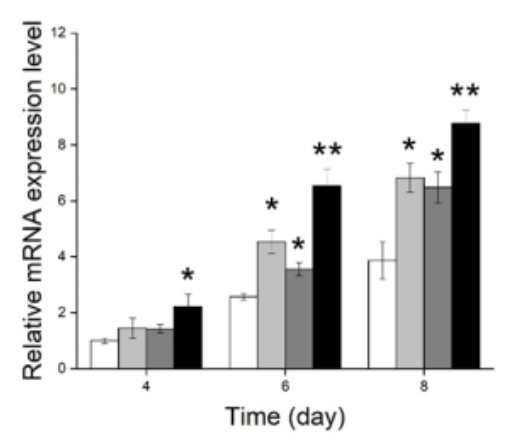

B

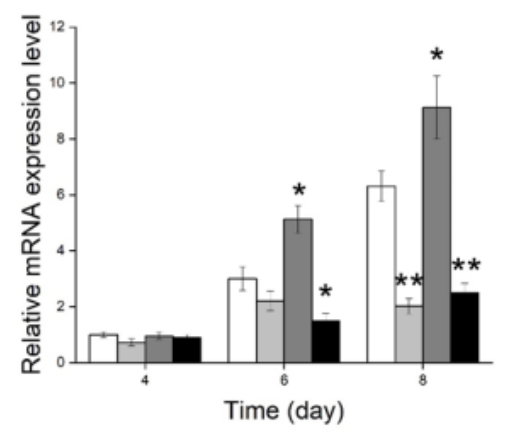

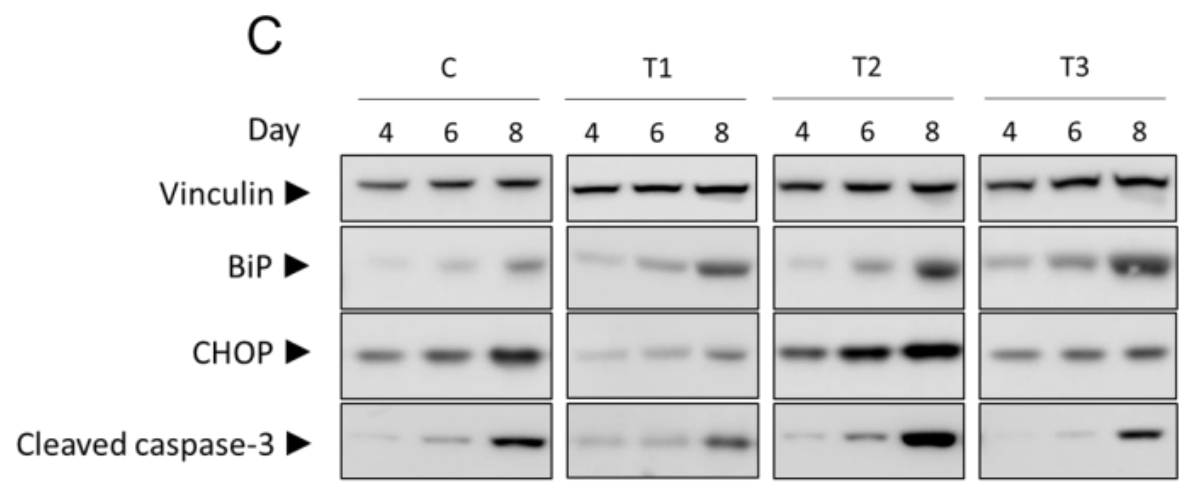

This article is protected by copyright. All rights reserved. 
Figure 4. Western blots of ATF6, XBP1s, eIF2 $\alpha$, p-eIF2 $\alpha$, calnexin, and GRP94 during shake flask cultures. Cells were harvested on day 4, 6, and 8 of the cultures shown in Figure 2. Vinculin was used as a loading control. No chemical reagent (C), BIX only (T1), DMSO only (T2), and BIX and DMSO (T3).

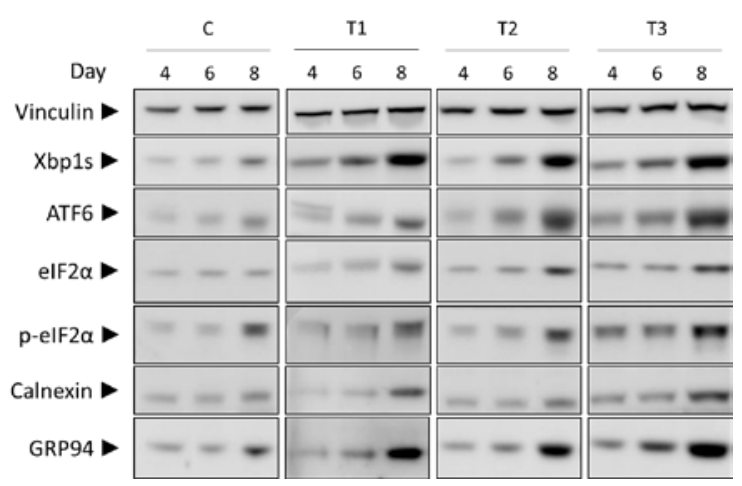

Figure 5. (A) Profiles of mAb galactosylation during shake flask cultures. Culture supernatants were harvested on days 6 and 9 of the cultures shown in Figure 2. No chemical reagent on day 6 (white), BIX and DMSO on day 6 (dashed white), no chemical reagent on day 9 (light gray), and BIX and DMSO on day 9 (dashed light gray). G0, G1, and G2 represent G0 + G0F, G1F + G1S1F, and G2F + G2S1F + G2S2F, respectively. (B) qRT-PCR of B4galt1, B4galt2, B4galt3, Gla, and Glb1 during shake flask cultures. Cells were sampled on day 4, 6, and 8 of the cultures shown in Figure 2. Values were normalized to the control at day 4. No chemical reagent on day 4 (white), BIX and DMSO on day 4 (dashed white), no chemical reagent on day 6 (light gray), BIX and DMSO on day 6 (dashed light gray), no chemical reagent on day 8 (gray), and BIX and DMSO on day 8 (dashed gray). Error bars represent the standard deviations calculated from three independent experiments. $* P<0.05,{ }^{*} * P<0.01$.

A
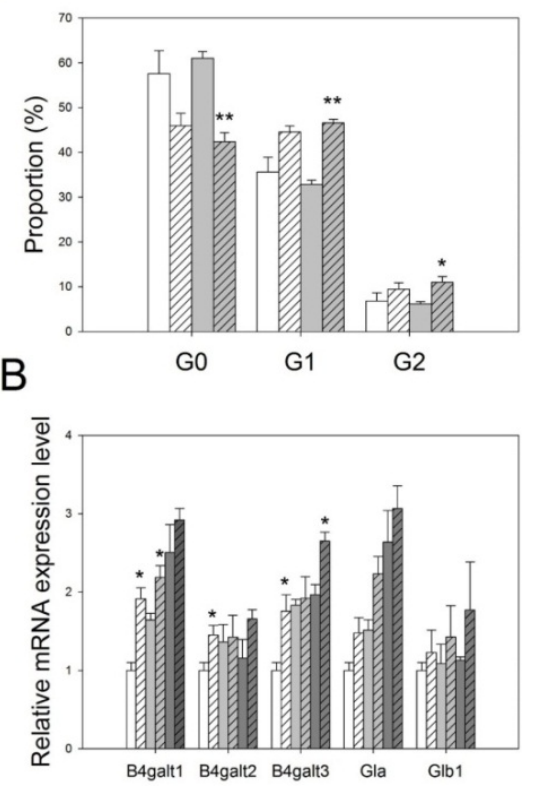

This article is protected by copyright. All rights reserved. 
Figure 6. Profiles of (A) cell growth, (B) viability, (C) mAb concentration, (D) mAb galactosylation during fed-batch cultures in a bioreactor with co-addition of BIX and DMSO. (A - C) No chemical reagent (black square) and $50 \mu \mathrm{M}$ BIX and 1\% v/v DMSO (black circle). (D) No chemical reagent on day 10 (white), $50 \mu \mathrm{M}$ BIX and 1\% v/v DMSO on day 10 (dashed white), No chemical reagent on day 14 (gray), and $50 \mu \mathrm{M}$ BIX and 1\% v/v DMSO on day 14 (dashed gray). G0, G1, and G2 represent G0 + G0F, G1F + G1S1F, and G2F + G2S1F + G2S2F, respectively. Error bars represent the standard deviations calculated from duplicate experiments. ${ }^{*} P<0.05$.

A

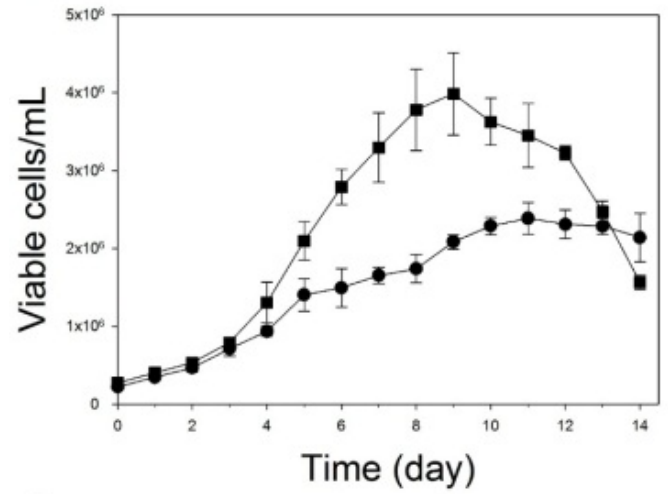

C

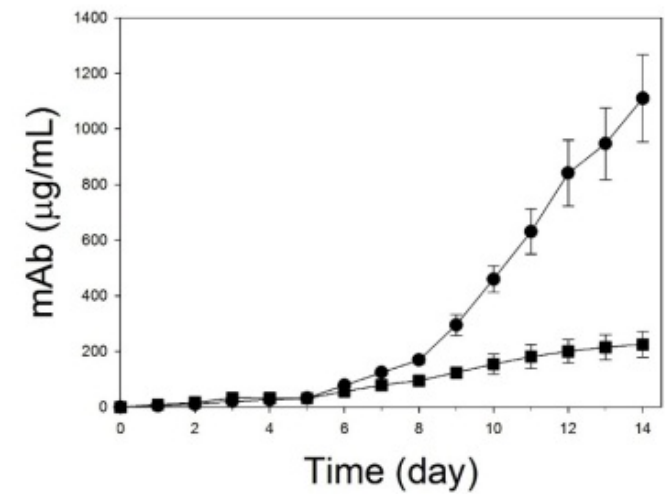

B

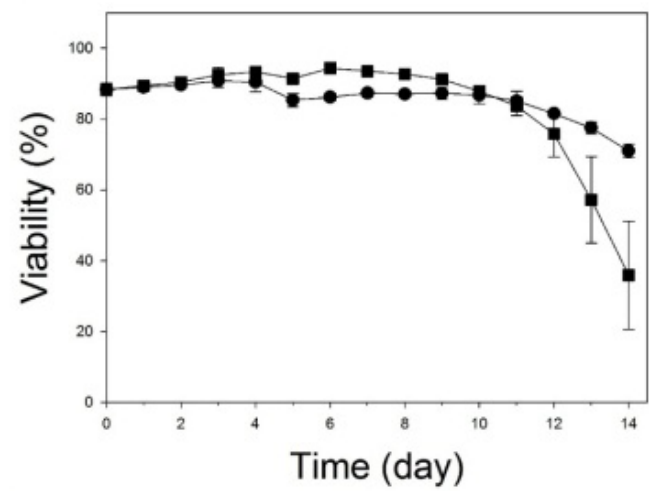

D

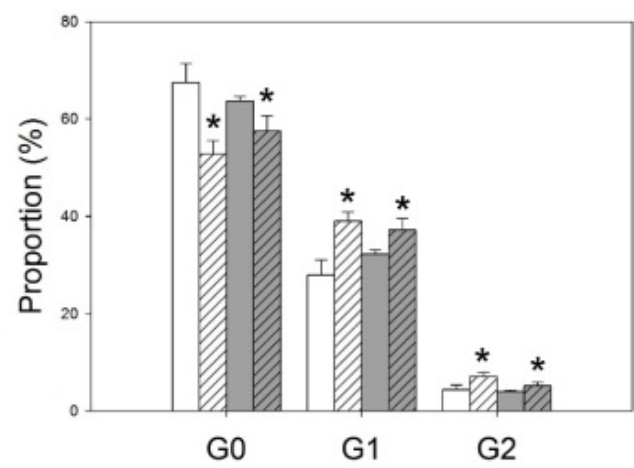

This article is protected by copyright. All rights reserved. 
Figure 7. Profiles of (A) cell growth, (B) viability, (C) mAb concentration, and (D) mAb galactosylation of CS13-1.00 cells during shake flask cultures with the coaddition of BIX and DMSO. (A - C) no chemical reagent (black square), $50 \mu \mathrm{M}$ BIX (black circle), $1 \%$ v/v DMSO (black up-triangle), and $50 \mu \mathrm{M}$ BIX and 1\% v/v DMSO (black down-triangle). (D) No chemical reagent on day 6 (white), $50 \mu \mathrm{M}$ BIX and 1\% v/v DMSO on day 6 (dashed white), no chemical reagent on day 9 (light gray), and 50 $\mu \mathrm{M}$ BIX and 1\% v/v DMSO on day 9 (dashed light gray). G0, G1, and G2 represent $\mathrm{G} 0$ + G0F, G1F + G1S1F, and G2F + G2S1F + G2S2F, respectively. Error bars represent the standard deviations calculated from three independent experiments. ${ }^{*} P$ $<0.05$

A

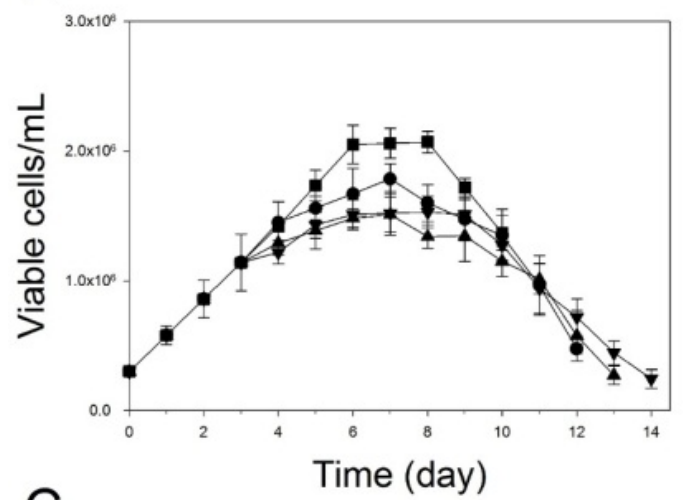

C

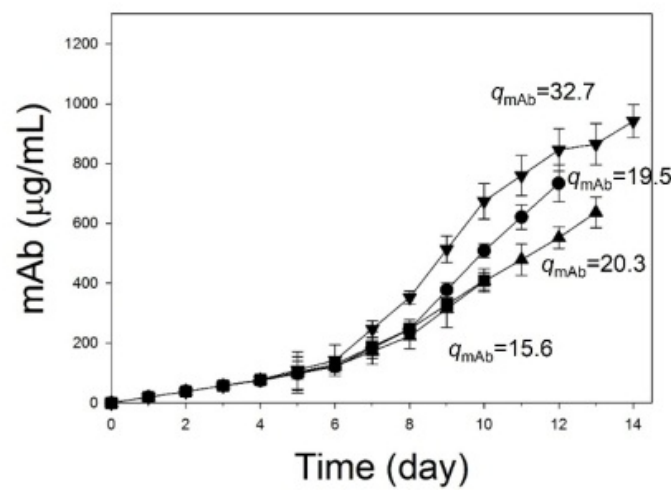

B

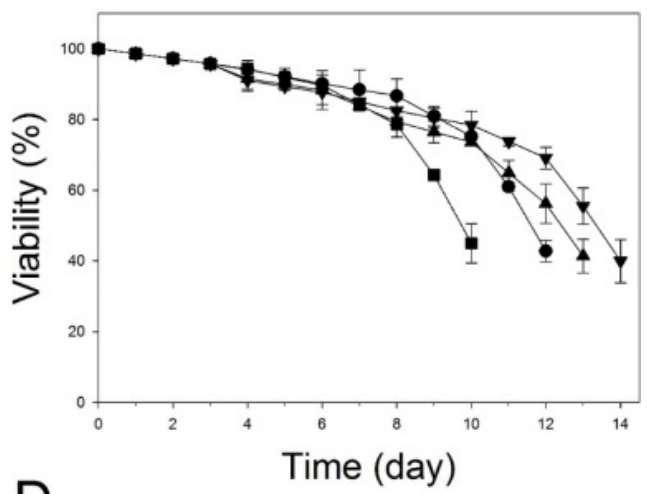

D

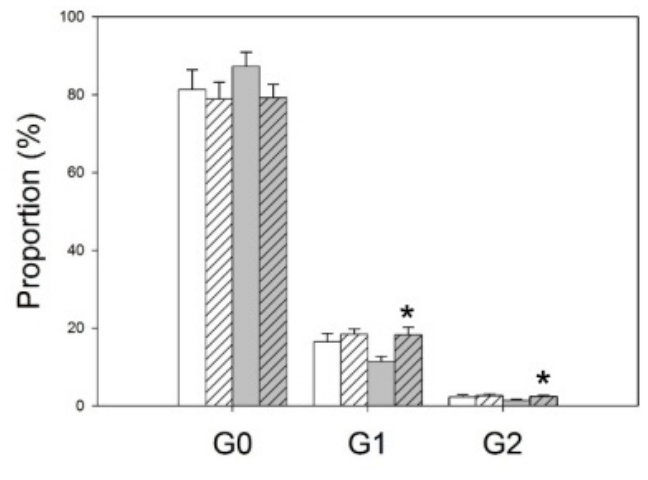

This article is protected by copyright. All rights reserved. 
Table 1. The $\mu$, MVCC, $q_{\mathrm{mAb}}$, MMC, and IVCC during shake flask cultures with the addition of BIX and/or DMSO. No supplements (C), $50 \mu \mathrm{M}$ BIX (T1), 1\% v/v DMSO (T2), and BIX and DMSO (T3). Values are means \pm standard deviations of three independent experiments.

\begin{tabular}{cccccc}
\hline \multicolumn{7}{c}{ MVCC } & & \\
& $\mu\left(\right.$ day $\left.^{-1}\right)$ & $\begin{array}{c}q_{\mathrm{mAb}} \\
\text { cells } / \mathrm{mL})\end{array}$ & $\begin{array}{c}\text { MMC } \\
(\mu \mathrm{g} / \mathrm{mL})\end{array}$ & $\begin{array}{c}\text { IVCC } \\
\left(10^{6} \text { cells } \cdot \text { day } / \mathrm{mL}\right)\end{array}$ \\
\hline $\mathrm{C}$ & $0.52 \pm 0.04$ & $4.75 \pm 0.13$ & $3.35 \pm 1.88$ & $114.3 \pm 22.6$ & $27.5 \pm 2.6$ \\
\hline T1 & $0.50 \pm 0.03$ & $4.37 \pm 0.24$ & $6.70 \pm 1.43$ & $283.8 \pm 40.0$ & $32.9 \pm 4.0$ \\
\hline T2 & $0.47 \pm 0.03$ & $3.55 \pm 0.43$ & $10.44 \pm 2.57$ & $525.3 \pm 96.2$ & $23.8 \pm 4.6$ \\
\hline T3 & $0.51 \pm 0.02$ & $4.15 \pm 0.15$ & $14.36 \pm 1.52$ & $1096.3 \pm 77.3$ & $30.9 \pm 2.4$ \\
\hline
\end{tabular}

Table 2. The $\mu$, MVCC, $q_{\mathrm{mAb}}$, MMC, and IVCC during fed-batch cultures in a bioreactor with the addition of BIX and DMSO. No supplements (C), and BIX and DMSO (T). Values are means \pm standard deviations of duplicate experiments.

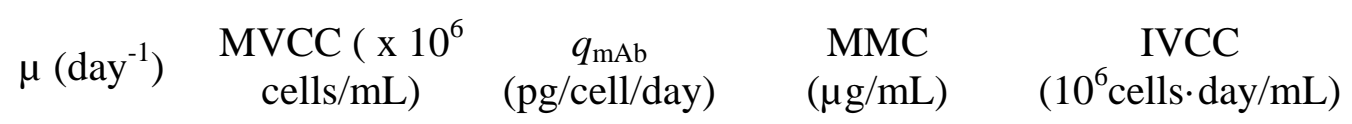

$\begin{array}{cccccc} & 0.41 \pm & & & & \\ \text { C } & 0.01 & 3.99 \pm 0.53 & 0.53 & 225.6 \pm 45.6 & 32.7 \pm 3.1\end{array}$

$\begin{array}{cccccc} & 0.37 \pm & 2.39 \pm 0.21 & 25.78 \pm & 1109.75 \pm & 21.4 \pm 1.9\end{array}$

This article is protected by copyright. All rights reserved. 